\title{
Migration of primordial germ cells to the developing gonadal ridges in the tammar wallaby Macropus eugenii
}

\author{
S. L. Ullmann* , G. Shaw, G. T. Alcorn ${ }^{\dagger}$ and M. B. Renfree \\ Department of Zoology. The University of Melbourne, Parkville, Victoria 3052, Australia
}

\begin{abstract}
Primordial germ cells (PGCs) of the tammar wallaby Macropus engenii have a distinctive morphology and stain positively for alkaline phosphatase. PGCs are identifiable in embryos with 12 somites, on about day 17 of the 26.5 day gestation period, when they are located in all three germ layers of the developing embryo and in the endoderm of the bilaminar and vascular (trilaminar) yolk sac membranes. PGCs are positive for alkaline phosphatase (ALP) at least between days 17 and 22 of pregnancy. In whole mounts on day 17, three groups of cells positive for ALP occur: about 40 just caudal to the neural tube, and about 20 distributed on either side of the last three somites. By day 21, there are about 150 PGCs in the newly formed gonadal ridges and 275 in the mesenteries. On days 21-22, there are PGCs in the umbilical mesoderm, the dorsal mesentery and the coelomic angles between the dorsal mesentery and the mesonephroi. On day 22, most ALP-positive PGCs are located in the dorsal mesentery, where they occur in groups. They apparently do not migrate through the hindgut endoderm, but occasional PGCs are seen in sites such as the mesonephros, the adrenals, the blood vessels of the yolk sac and in the vicinity of the dorsal aorta and dorsal nerve cord. Between day 23 and day 25, I day before birth, most of the 3200-4000 PGCs complete their migration to the gonadal ridges. Although there are marked differences between embryogenesis of tammars and mice, development and the pattern of migration of PGCs in this marsupial mammal are similar to that of eutherian mammals.
\end{abstract}

\section{Introduction}

The extra-gonadal origin of the primordial germ cells (PGCs) is well documented for many species (Nieuwkoop and Sutasurya, 1979, 1981), but their precise genesis within the embryos of higher vertebrates remains of great interest. In birds, the PGCs originate in the epiblast, mostly from the central disc region and then move to the extra-embryonal germinal crescent, returning to the embryo through the blood circulation after gastrulation (Ginsburg and Eyal Giladi, 1986, 1987; Ginsburg, 1994, 1996).

The most detailed studies in mammals of the origin and migration of the PGCs are in the mouse. In elegant experiments using chimaeras, Gardner (1978) first established that the PGCs originate in the epiblast. This has been confirmed by Lawson and Hage (1994) who have further shown, by clonal analysis, that the PGC precursors form part of the extra-embryonic mesoderm and are not restricted by lineage while in the epiblast. The founding population of PGCs comprises about 45 cells, which become established at the mid-primitive streak

Permanent addresses: *Division of Environmental and Evolutionary Biology, Institute of Biomedical and Life Sciences, University of Glasgow, Glasgow G12 8QQ, UK; and

*University of Western Sydney Macarthur, PO Box 555, Campbelltown, NSW 2560. Australia.

*Correspondence.

Received 13 August 1996 stage. The PGCs then migrate to the developing gonadal ridges during fetal life (McLaren, 1981). Thus, the germ-cell lineage is determined early in development; however, while somatic cells become progressively more restricted in potency during development, gametogenesis results in the reacquisition of pluripotency (Wei and Mahowald, 1994).

Migrating PGCs are characterized by their large heterochromatic nuclei, their clear cytoplasm and, in most mammals investigated, their alkaline phosphatase (ALP) content. Snow (1981) observed PGCs in the tissues at the base of the allantois at about 7 days postcoitum. More recently, Ginsburg et al. (1990) used whole mounts of presomite embryos 7-7.25 days postcoitum to demonstrate a cluster of ALP-positive cells in the extra-embryonic mesoderm just posterior to the primitive streak. By 8 days these cells, numbering about 125, were sited at the base of the allantois and in the hindgut endoderm locations typical for PGCs. These authors noted the appearance of an intensely staining ALP spot in the putative PGCs during development and speculated that this could be a maturation phenomenon.

During the next 5 days the PGCs migrate to the gonadal ridges via the wall of the invaginating hindgut and the dorsal mesentery. As they migrate the number of PGCs increases rapidly by mitotic divisions to about 26000 by day 13.5 (Tam and Snow, 1981). In humans, a similar sequence of events occurs. PGCs are first recognized in the yolk-sac stalk on day 24 of pregnancy as ALP-positive cells and they migrate to the 
gonadal ridges via the dorsal mesentery (Byskov and Hoyer, 1994). At the onset of meiotic arrest in the female, the germ cells lose their reaction for ALP.

Despite the current interest in PGCs of eutherian mammals, those of marsupial mammals have received scant attention, although marsupials are born at a developmental stage equivalent to that of a fetal eutherian. The first descriptions were of PGCs in the fetus of the tammar wallaby Macropus engenit (Alcorn, 1975) and in fetuses of the bandicoots Isoodon macrourus and Perameles nasuta (Ulimann, 1981). In most eutherians, germ cells have completed their migration and their numbers are declining by birth (Byskov and Høyer, 1994). This is in sharp contrast to the situation in marsupials (Tyndale-Biscoe and Renfree, 1987): for example, in bandicoots, the PGCs are still migrating at birth (Ullmann, 1981, 1989); while in the tammar female germ cells reach peak numbers only at about 50 days postpartum, progressively switching from mitotic to meiotic division between day 25 and day 50 (Alcorn and Robinson, 1983). This reflects the altricial nature of the marsupial neonate, which results in much of sexual differentiation occurring postnatally.

Marsupials may be especially suitable for investigations of the origin and migration of the PGCs because the embryo develops on the surface of a vesicle, unencumbered by an egg cylinder as in the mouse. This study gives a detailed description of the characteristics of PGCs and their migration to the gonadal ridges in the tammar wallaby in embryos from soon after the time of gastrulation to full-term fetuses, which encompasses days 17-26 of the 26.5 day gestation period.

\section{Materials and Methods}

\section{Animals}

Tammar wallabies (Macropus engenii) of Kangaroo Island origin were obtained from our breeding colony maintained at Monash University as previously described (Renfree et al., 1989). Six fetuses were obtained from tammars in a colony maintained at Macquarie University. Pregnancies were initiated during lactational quiescence by removal of pouch young (RPY) from females known to be carrying diapausing blastocysts. The day of RPY was designated day 0 . The gestation period after RPY is $26.4 \pm 1.0$ days (Tyndale-Biscoe and Renfree, 1987).

Embryos and fetuses were removed from the uterus 17 $(n=7), \quad 18 \quad(n=4), 19 \quad(n=2), 20 \quad(n=4), 21 \quad(n=11), 22$ $(n=14), 23(n=11), 24(n=7), 25(n=10)$ or $26(n=4)$ days after RPY, as previously described (Renfree and TyndaleBiscoe, 1973, 1978), after the mother had been killed with an overdose of sodium pentobarbitone (Abbott Laboratories, Kurnell, NSW) in $0.9 \%(\mathrm{w} / \mathrm{v})$ saline. To accommodate variation in embryonic development on a given day of RPY, or to age fetuses of uncertain gestation, embryonic and fetal stages were normalized using the growth curves for the tammar reported by Renfree and Tyndale-Biscoe (1973) and Tyndale-Biscoe and Renfree (1987). Using these curves, four specimens of unknown gestation dates were aged at day 20,21, 22 and 25. Sex was determined by karyotyping the head tissue of the fetus, or by direct observation of the presence of scrotal or mammary primordia (O et al., 1988).
Care and treatment of the animals conformed to the National Health and Medical Research Council guidelines (National Health and Medical Research Council of Australia, 1990) and all experiments were approved by Institutional Animal Experimentation Ethics Committees.

\section{Light microscopy}

Embryos and fetuses were fixed by immersion in either neutral, buffered, 10\% formalin or Bouin's fixative, dehydrated through a graded ethanol series, cleared in Histoclear (Interpath Services, West Heidelberg, Victoria) and embedded in paraffin wax at $56^{\circ} \mathrm{C}$. Serial sections were cut at $7 \mu \mathrm{m}$ or $10 \mu \mathrm{m}$ intervals and stained with Ehrlich's haematoxylin and alcoholic eosin. The six fetuses from the Macquarie University colony were used for counting numbers of germ cells. They were fixed in Bodian's fluid, wax embedded, sectioned at $10 \mu \mathrm{m}$ and stained with Harris' haematoxylin and eosin.

\section{Alkaline phosphatase staining}

For the demonstration of ALP, fetuses at day $22(n=3)$ were placed in OCT 4583 (Miles Diagnostics, Elkhart, IN) cryomounting compound and frozen over a slurry of dry ice in absolute ethanol. Serial frozen sections were cut at either $7 \mu \mathrm{m}$ or $10 \mu \mathrm{m}$ intervals on a Reichert Ultracut freezing microtome at $-20^{\circ} \mathrm{C}$ and transferred onto glass slides. Sections were stained with Fast-Blue BB salt ( $1 \mathrm{mg} \mathrm{ml}{ }^{-1}$; Gurr, BDH Chemical Ltd, Poole) and naphthol AS phosphate sodium salt (1 $\mathrm{mg} \mathrm{ml}^{-1}$; Sigma Chemical Co., St Louis, MO) in Tris buffer $\left(0.2 \mathrm{~mol} \mathrm{l}^{-1}\right.$; $\mathrm{pH}$ 9.4) that was made up immediately before use and filtered on to the slides. They were stained at room temperature in the dark for either 7 or $15 \mathrm{~min}$. The shorter time reduced background staining. Sequential sections were stained with haematoxylin and eosin, left unstained or counterstained lightly in neutral red.

Vesicles with somite-stage embryos $17(n=2)$ or $18(n=1)$ days after RPY were incubated whole in the Fast-Blue staining solution immediately after they were taken from the uterus, and then photographed. The embryos at day 17 were submerged and then frozen in OCT 4583, as described above, and sectioned on the cryostat. The embryo at day 18 was fixed in neutral, buffered, $10 \%$ formalin, embedded in wax and serially sectioned.

\section{Morphometric analyses}

In the six fetuses obtained from the Macquarie colony, camera lucida drawings were made of approximately every tenth to fifteenth section (depending on the size of the embryo) of the caudal half of each embryo. Sections were examined to identify the PGCs, which were then counted and their positions plotted on the camera lucida drawings. Scale drawings of the urogenital systems of each embryo were prepared from the camera lucida outlines and the approximate positions of the PGCs were plotted on these drawings. The numbers of PGCs were estimated by the method of Abercrombie (Abercrombie, 1946; Wreford, 1995). 

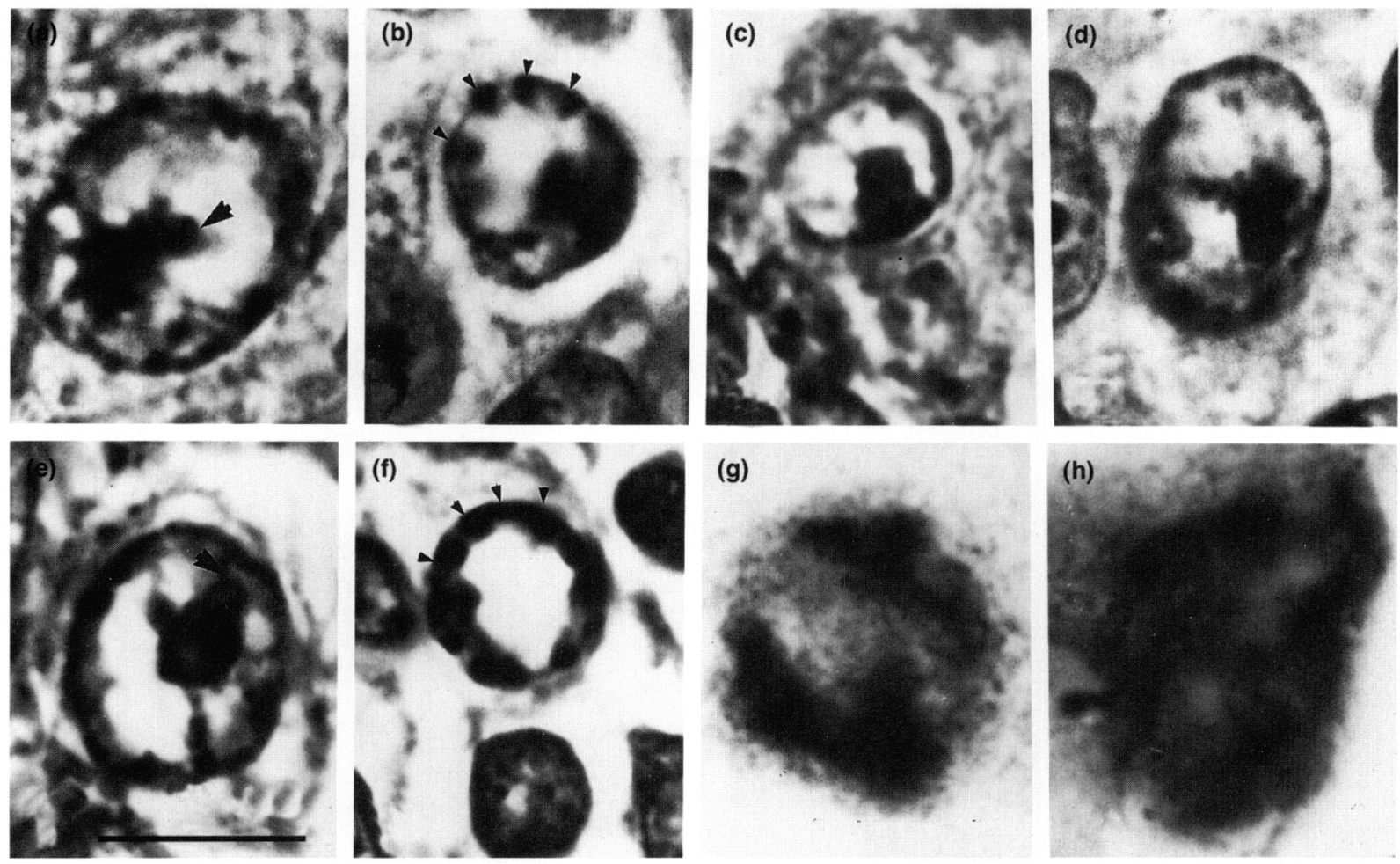

Fig. 1. Morphology of the primordial germ cells (PGCs) of male and female tammars in different locations. Migrating PGCs frequently show prominent chromatin beading (small arrowheads) and nucleolar-associated heterochromatin masses (large arrowhead) but these characteristics are not associated with sex or location. (a) Male and (b) female on day 21 in coelomic angles; (c) male on day 23 and (d) female on day 21 in dorsal mesentery; (e) male on day 23, in gonad; ( $)$ female on day 21 in coelomic angle; ( $\mathrm{g}$ ) a single, and $(\mathrm{h})$ a pair of PCGs on day 22, stained for alkaline phosphatase (ALP). The nucleus is not ALP positive and shows as a clear region. Scale bar represents $10 \mu \mathrm{m}$.

\section{Results}

\section{Identification of PGCS}

Morphology of PGCs. The tammar PGCs are similar in appearance to those of other mammals (Fig. 1). They are large compared with somatic cells and have a large, vesicular nucleus that can be circular, subspherical or lobed in sections. They have a prominent nucleolus, a well-defined nuclear envelope that often appears to be beaded with chromatin and that may have eosinophilic chromatin threads between it and the nucleolus. PGCs have a relatively pale-staining nucleoplasm and cytoplasm that is agranular. The cells are ovoid, sometimes pear-shaped and the cell membrane is usually difficult to see. Occasionally, pseudopod-like extensions can be seen.

No sexual dimorphism could be found in the cytology or in the arrangement of the nucleolus-associated heterochromatin in the migrating PGCs or in PGCs in the gonads (Fig. I).

Alkaline phosphatase staining. Tammar PGCs were found to be ALP-positive (Figs 1 and 2). Although this enzyme is not exclusive to PGCs, ALP-positive cells were located in the bilaminar yolk-sac membrane, along their expected route of migration as well as in ectopic sites. The reaction was localized to the cytoplasm (Fig. Ig, h). ALP staining not associated with PGCs also occurred in several locations in frozen sections. Staining was strong in the mesonephros and less intense in the dorsal nerve cord, the gonadal stroma and in the mesenchyme beneath the dorsal aorta. Diffuse staining was seen in the region of the sinus terminalis and in the trilaminar yolk sac.

\section{Migration of $P G C_{s}$}

On day 17 , most vesicles had reached a diameter of $15 \mathrm{~mm}$ and the embryos were at the 12-14-somite stage (Fig. 2a). ALP-positive cells could be identified as groups of 20 individual cells located adjacent to the last three somites and as 40-50 cells around the caudal end of the neural tube (Fig. $2 \mathrm{~b}$ ) in whole mounts. In sections of embryos at days 17 and 18 (Fig. 3), the PGCs were found in the neural plate, within and between the embryonic ectoderm, mesoderm and endoderm as well as in the endoderm of the bilaminar and trilaminar yolk sac. Some PGCs were also found in the ectoderm at the junction of the bilaminar and trilaminar yolk sacs.

The gonadal ridges began to develop between 20 and 21 days after RPY. Although a few PGCs were present in the posterior of the ridges and around the mesothelium of the coelomic angles, the majority were found in the dorsal mesentery, posterior to the ridges (Fig. 4). The peak of PGC migration occurred on days 21 and 22 , when the crown-rump length of most fetuses was $8-10 \mathrm{~mm}$. On day 21 , about 275 PGCs were located in the central region of the dorsal 


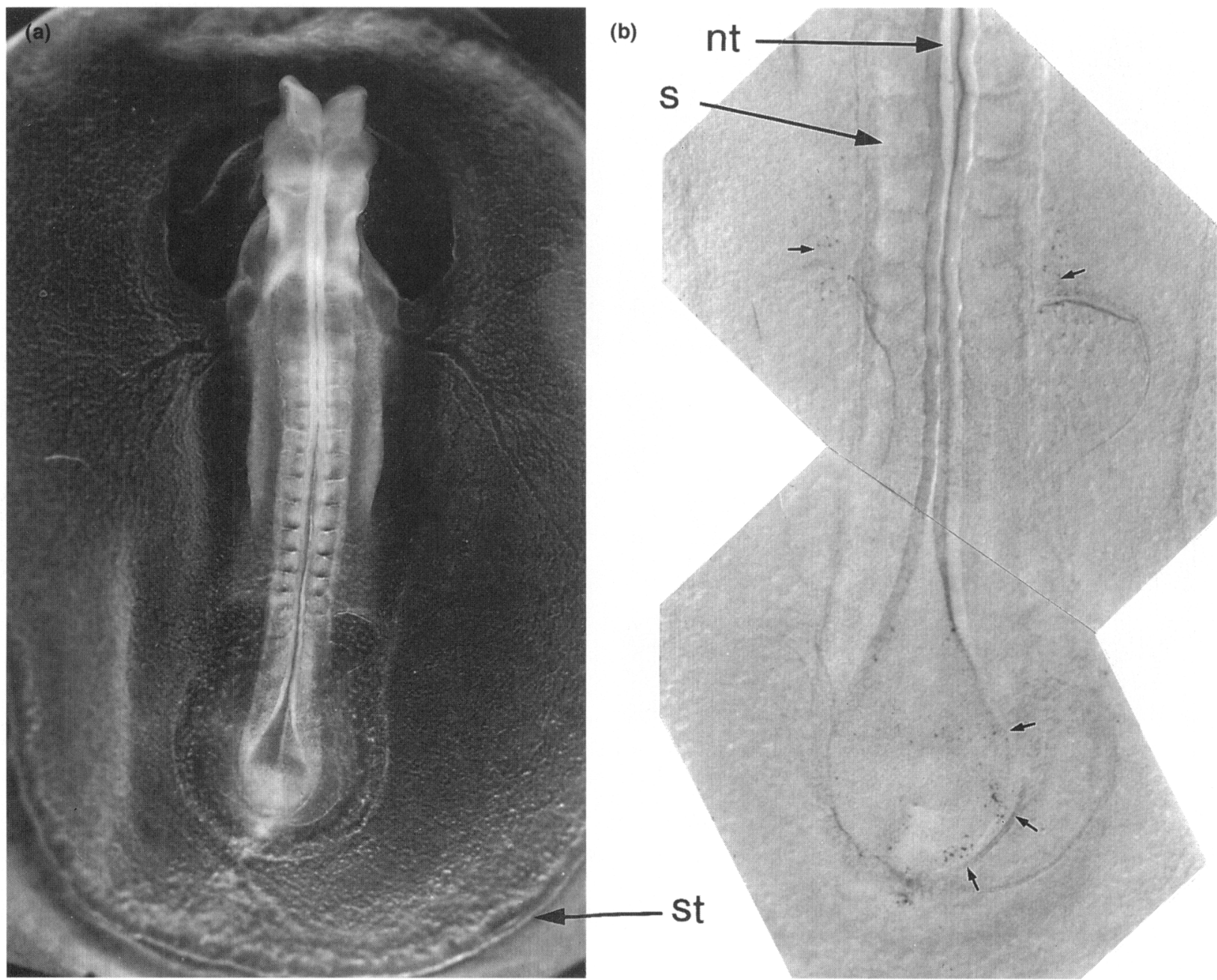

Fig. 2. Distribution of primordial germ cells (PGCs) in a whole mount of the tammar embryo on day 17 after removal of pouch young (RPY). (a) Embryo cut from the vesicle just beyond the margin of the sinus terminalis (st). The vascular area is prominent. The neural tube is still open posterior to the brain and somites are clearly visible. (b) Enlargement showing the distribution of PGCs identified by alkaline phosphatase (ALP) staining in two areas lateral to the third last somite (s) and the third area centrally close to the regressing primitive streak (small arrows); nt, neural tube.

mesentery (Fig. 4). There were fewer (about 150) in the gonad primordia, mostly in the caudal half (Figs 5 and 6). PGCs were also observed in the yolk-sac membranes, the mesoderm of the umbilical hernia, the coelomic angles (Figs 4 and 7), the mesenchyme below the aorta and between the mesonephros and gonadal ridges.

By day 22, PGCs were present throughout the length of the gonadal ridges, although there were more PGCs in the dorsal mesentery (Fig. 6c). As in the ridges, the mass of migrating PGCs had shifted more anteriorly within the mesentery (Fig. 6). ALP-positive PGCs were observed singly or in interconnected groups (Fig. 4c) and were especially numerous in the dorsal mesentery and in the coelomic angles adjacent to the developing gonadal ridges. ALP-positive cells were also observed in the membrane of the bilaminar yolk-sac the umbilical mesoderm, below the aorta and, on one occasion, within a blood vessel of the vascular yolk sac (Fig. 7b, c).

After day 23, PGCs were increasingly located within the gonadal ridges where they were more evenly distributed than before. Those still in the dorsal mesentery and skirting the coelomic angles were mostly at the mesonephroi, but more laterally than previously, and there were fewer present in the mesenchyme above the gonadal ridges (Fig. 6d). By day 24 there were clearly more PGCs within the gonadal ridges than the dorsal mesentery (Figs. 6e).

Some PGCs appeared to migrate cranial to the gonad primordia within the dorsal mesentery and miss their destination. Such misplaced or ectopic PGCs were observed in the mesonephros (Fig. 7e), the adrenal primordium (Fig. 7f) and in the vicinity of the dorsal nerve cord and the aorta (Fig. 7b). In one fetus only, two PGCs were found in the hindgut endoderm (Fig. 7a).

By days 25 and 26, almost all (around 3200-4000) PGCs had reached the gonads (Fig. 6) while only 50 remained in the mesentery. The majority of PGCs were concentrated in the centres of the gonadal ridges, with their numbers decreasing anteriorly and posteriorly. There were only a few migrating PGCs in the dorsal mesentery (Fig. 6f). 

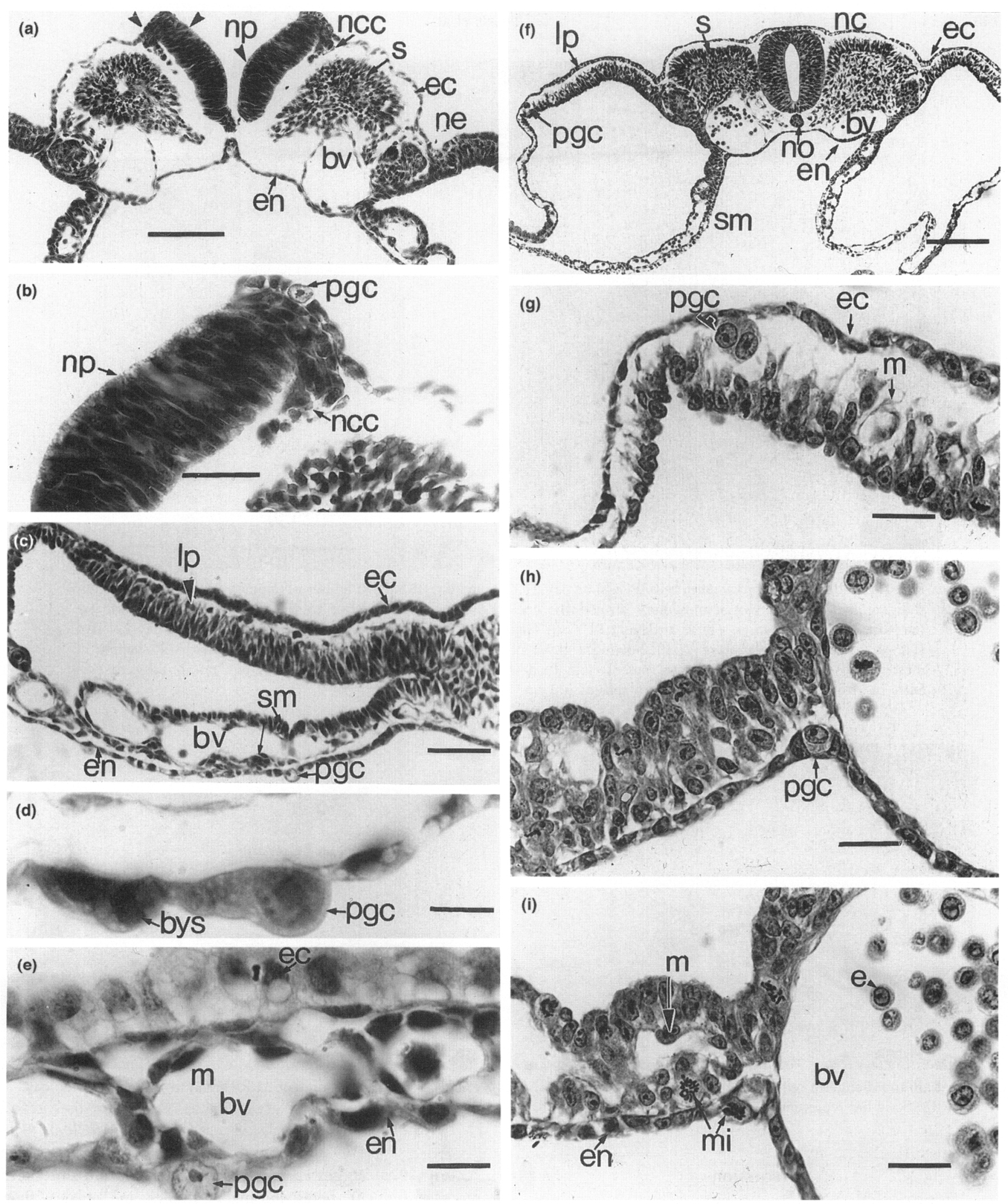

Fig. 3. Distribution of primordial germ cells (PGCs) in a 14-somite embryo of the tammar on day 17 after removal of pouch young (RPY) and in a 20-somite embryo on day 18 RPY. Transverse sections through embryos on day 17 (a-e) and day 18 (f-i). (a) Open neural tube showing neural crest cells separating from neural plate. Scale bar represents $100 \mu \mathrm{m}$. (b) High power of part of (a) showing PGCs within neurectoderm. Scale bar represents $30 \mu \mathrm{m}$. (c) Section through the lateral region showing a PGC protruding from the endoderm. Scale bar represents $50 \mu \mathrm{m}$. (d) A high power of a primordial germ cell in the endoderm of the bilaminar yolk sac showing the characteristic chromatin beading around the nuclear envelope. Scale bar represents $10 \mu \mathrm{m}$. (e) A PGC in the endoderm of the trilaminar yolk sac. Scale bar represents $15 \mu \mathrm{m}$. ( $\mathrm{f}$ ) An embryo at day 18 showing the neural tube. Scale bar represents $100 \mu \mathrm{m}$. (g) Enlargement of part of (f) showing PGCs between the ectoderm (ec) and the lateral plate mesoderm. Scale bar represents $50 \mu \mathrm{m}$. (h) PGC in the endoderm. Scale bar represents $50 \mu \mathrm{m}$. (i) Section adjacent to (h) showing two large mitotic cells, one in the endoderm and one in the mesoderm which are probably PGCs in division. Scale bar represents $50 \mu \mathrm{m}$. bv, blood

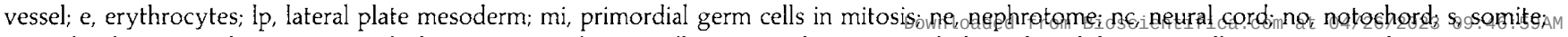
$\mathrm{sm}$, splanchnic mesoderm; np, neural plate; ncc, neural crest cells; ec, ectoderm; en, endoderm; bys, bilaminar yolk sac; m, mesoderm. free access 

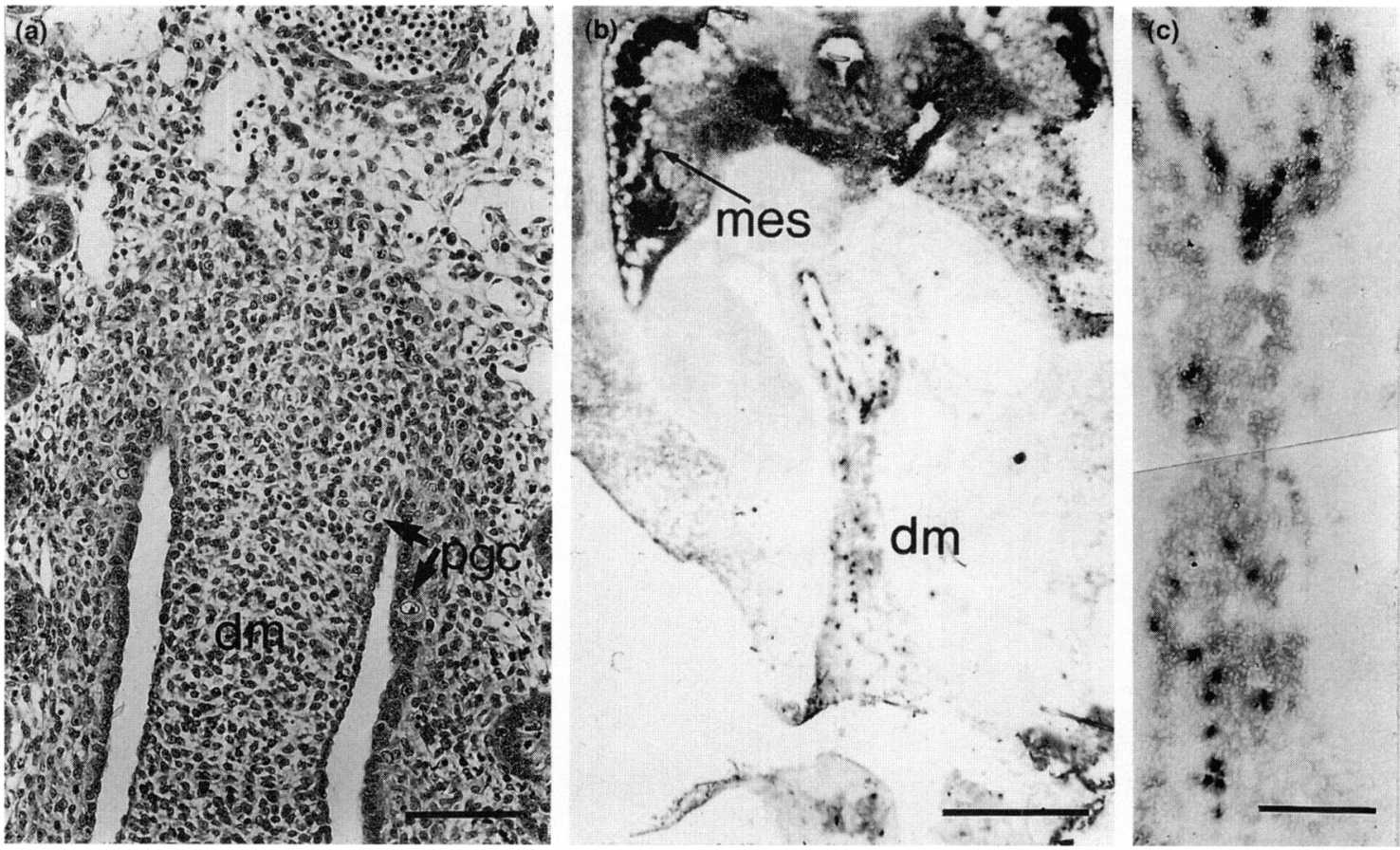

Fig. 4. Location of primordial germ cells (PGCs) on day 21 RPY and day 22 RPY in fetuses of tammar. (a) Transverse section showing PGCs in the dorsal mesentery around the coelomic angle and posterior to the gonadal ridge beneath the transformed mesothelium in a fetus at day 21 RPY. (b) Transverse cryostat section of the gonadal region of a day 22 fetus stained for alkaline phosphatase (ALP) showing a group of ALP-positive (dark stained) PGCs. Note also ALP staining in mesonephros. (c) High power of dorsal mesentery in (b). dm, dorsal mesentery; mes, mesonephros; pgc, primordial germ cell. Scale bars represent (a) $100 \mu \mathrm{m}$, (b) $1 \mathrm{~mm}$ and (c) $100 \mu \mathrm{m}$.
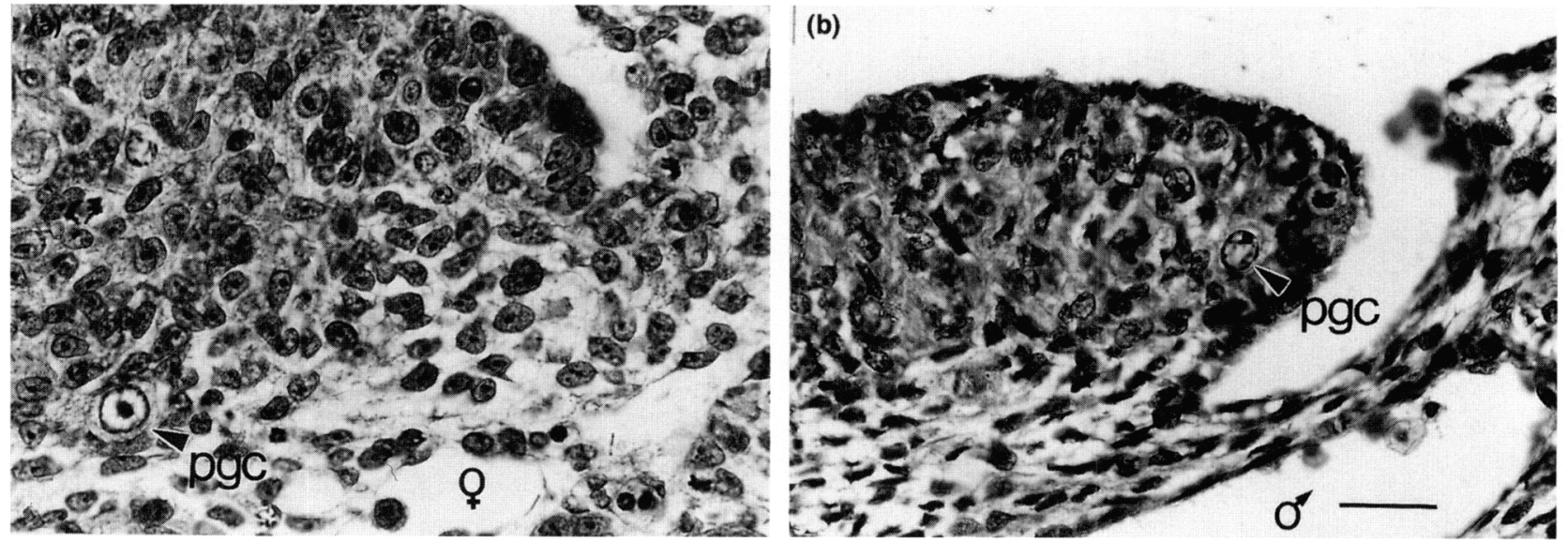

Fig. 5. Primordial germ cells (PGCs) in the gonadal ridge of (a) a female fetus of the tammar on day 21 RPY and (b) a male fetus on day 24 RPY. Scale bar represents $20 \mu \mathrm{m}$.

\section{Discussion}

Primordial germ cells are readily identifiable in tammar wallaby embryos and fetuses from at least day 17 (early-somite stage) of gestation through to term and they have a distinctive morphology that is similar to that of eutherian mammal PGCs. In contrast to the bandicoots (Ullmann, 1981) and the grey short-tailed opossum Monodelphis domestica (Maitland and Ullmann, 1993), they are ALP-positive. Tammar PGCs also have noticeable nucleolus-associated heterochromatin bodies.
Alcorn (1975) suggested that migrating PGCs have larger and more prominent heterochromatin bodies in females than in males, representing the contracted and hence inactive $X$ chromosome. Although we used more specimens in our study than Alcorn, we could not confirm any sexual dimorphism in migrating PGCs.

At the earliest stages examined (17 and 18 days), PGCs were found in all three embryonic germ layers and in the extraembryonic tissues. PGCs are initially more numerous in the ectoderm, but are frequently located between the ectoderm, 
(a)

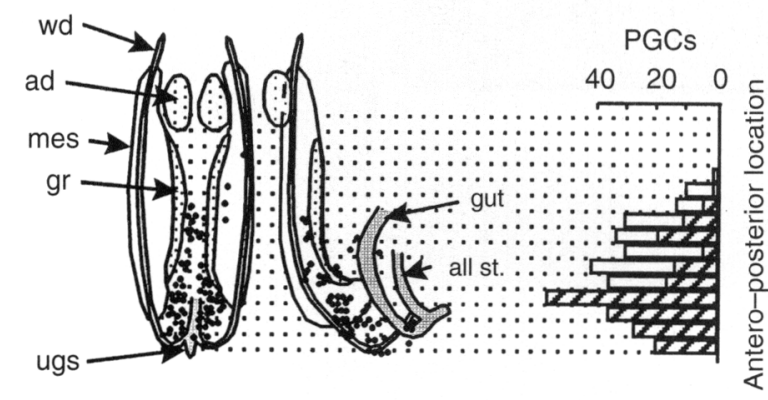

(b)

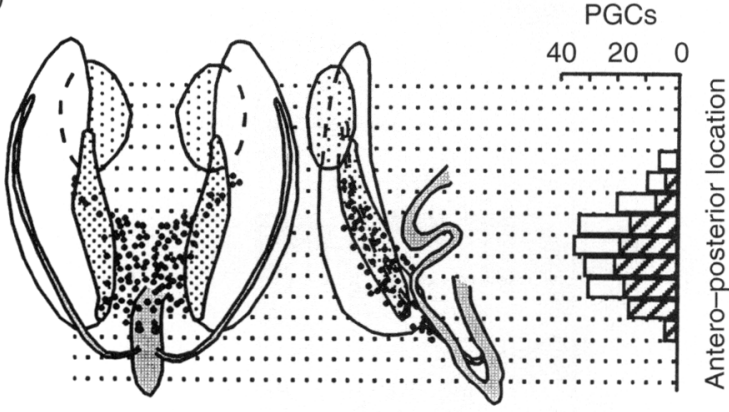

(c)

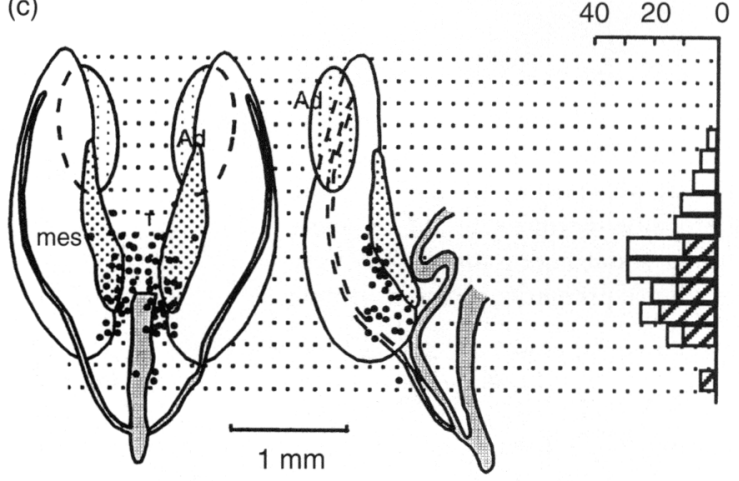

Ventral view

Right sagittal view (d)

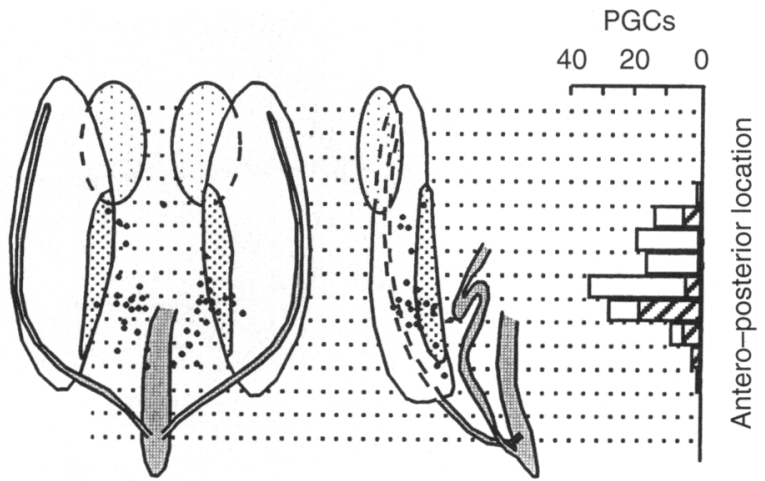

(e)
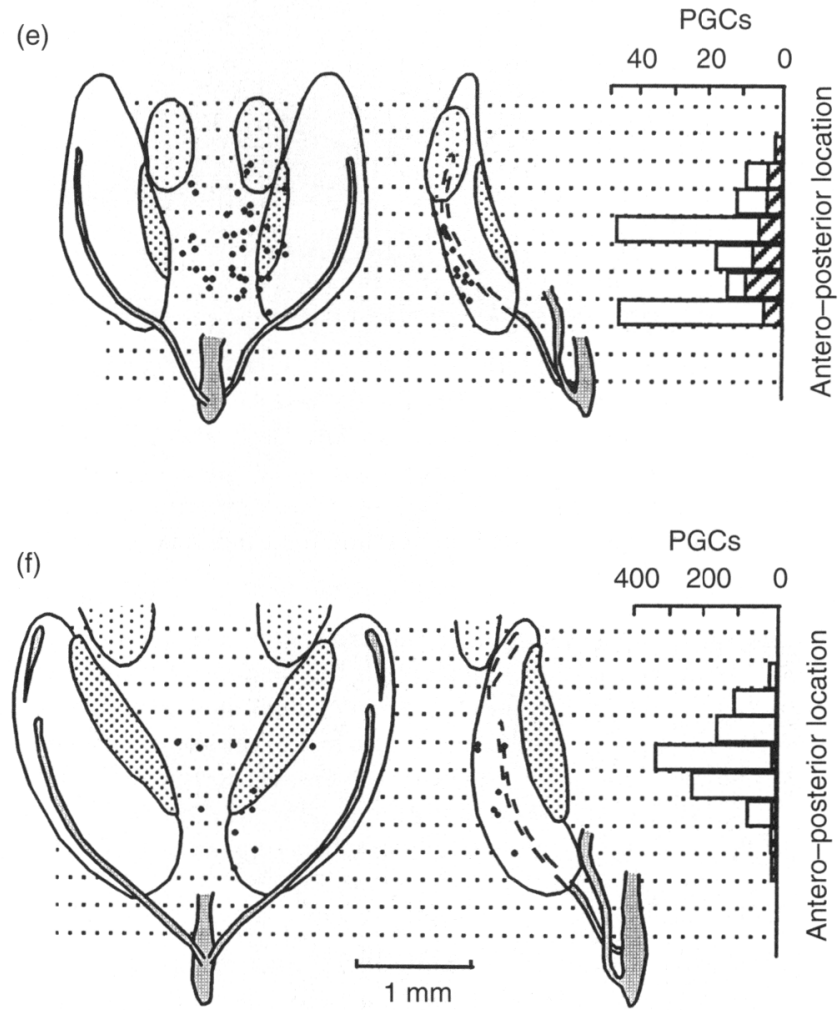

Ventral view

Right sagittal view

Fig. 6. Graphic reconstruction (all to the same scale) in ventral and right sagittal views of urinogenital systems of fetal tammars showing the approximate location of migrating primordial germ cells (PGCs) (dots) and relative numbers (histograms) of PGCs migrating (hatched bars) and in the gonadal ridges (open bars). (a) Male fetus at day 20 after removal of pouch young (RPY), (b) female fetus at day 21 RPY, (c) male fetus at day 22 RPY (d) female fetus at day 23 RPY, (e) female fetus at day 24 RPY, (f) male fetus at day 25 RPY. ad, adrenal; all. st., allantoic stalk; gr, gonadal ridge; mes, mesonephros; ugs, urogenital sinus; wd, wolffian duct.

mesoderm and endoderm. At later stages, they are absent from the ectoderm and are progressively more numerous in the endoderm. The wide distribution of PGCs in these early-somite embryos is quite unusual and warrants further study.

In mice, the PGCs migrate to the hindgut endoderm and mesoderm 9-10 days after coitus and reach the gonadal primordia 10.5-12.5 days after coitus via the dorsal mesentery (Ginsburg et al., 1990). In the tammar wallaby, the period of migration is more protracted than in the mouse, since PGCs were located in the dorsal mesentery and the gonadal primordia from day 21 (the peak of migration) until birth on day 26.5
RPY. In tammars, most PGCs appear to migrate into the fetus via the mesoderm in the umbilical region but, in contrast to the mouse, they were never observed in the allantois. Indeed, PGCs can be identified in the 12-somite embryo at day 17 , although the allantois does not form until after day 20 in the tammar (Renfree 1973; Renfree et al., 1996). The primary route of migration was via the splanchnic mesoderm around the hindgut and into the mesentery suspending it. They reached the gonadal ridges by skirting the coelomic angles. PGCs were only identified in the hindgut in one specimen although this is part of the principal migratory pathway not only in eutherians 

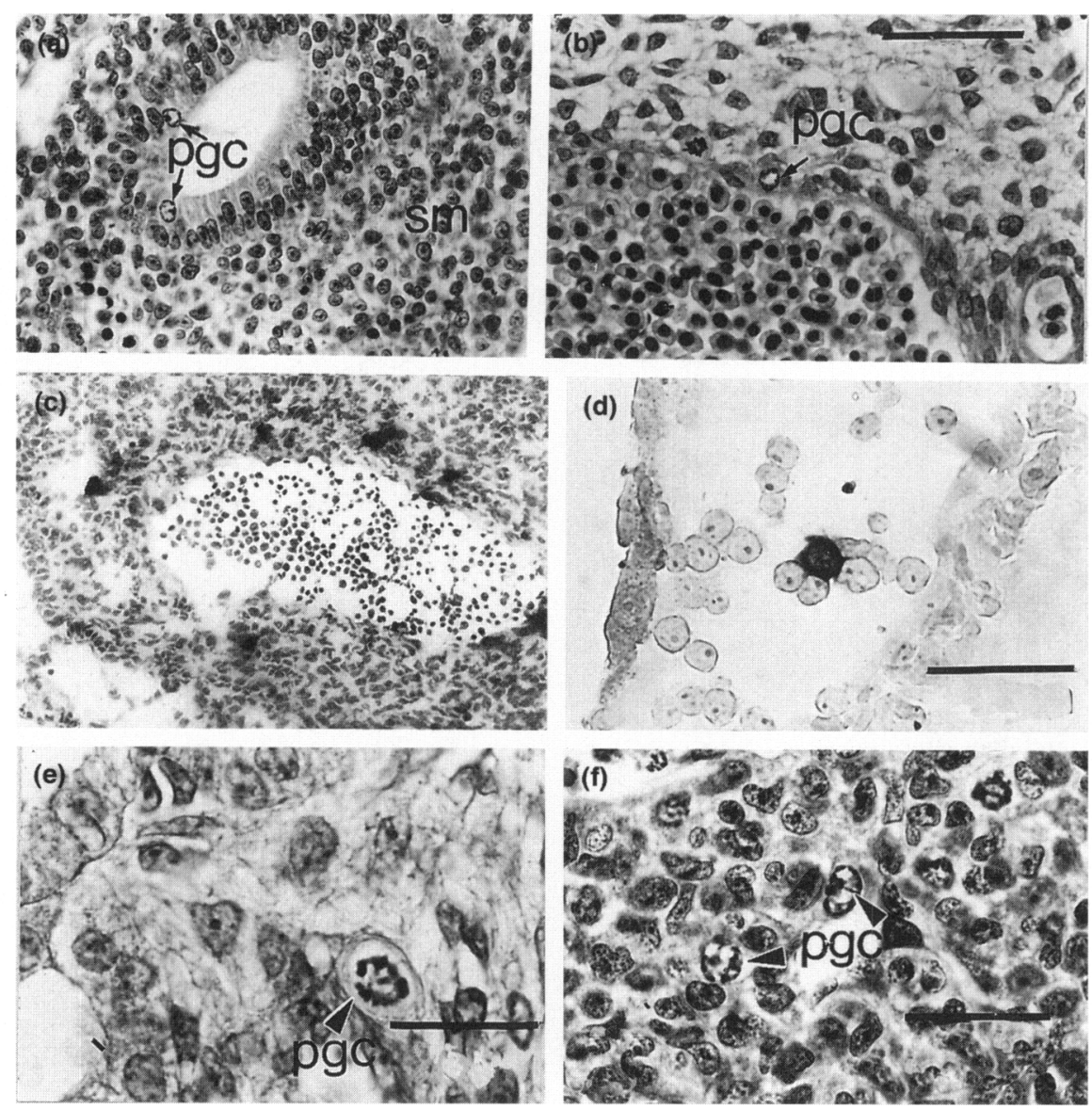

Fig. 7. Primordial germ cells (PGCs) in atypical sites of fetuses of tammars. (a) Transverse section through the hindgut of a fetus at day 2I RPY with two PGCs (arrows). (b) PGC in connective tissue around the aorta. (c) PGCs positive for alkaline phosphatase (AP) skirting the dorsal aorta at day 22. The somatic cells are counterstained to show erythrocytes. (d) A single ALP-positive cell thought to be a PGC in the centre of the blood vessel among the erythrocytes. (e) PGCs in the mesonephros on day 21 RPY. (f) PGC in the adrenal primordium. sm, splanchnic mesoderm. Scale bars represent: (a-d) $50 \mu \mathrm{m}$; (e, f) $25 \mu \mathrm{m}$.

but also in bandicoots (Ullmann, 1981) and apparently in the grey short-tailed opossum (Maitland and Ullmann, 1993). On the other hand, in the brushtail possum (Trichosurus vulpecula), as in the tammar, PGCs have not, so far, been reported in the hindgut, although they occur in the dorsal mesentery (Ullmann, 1993).

This difference between bandicoots and opossums, and tammars and possums, in the use of the hindgut by migrating PGCs may reflect differences in the timing of gonadal and gut development. Bandicoots and opossums are born at a relatively earlier stage of development than tammars and possums (grade 2 rather than grade 3, as defined by Hughes and Hall, 1988) but at birth still need a functional digestive tract, so gut development in bandicoots and opossums is probably advanced relative to the timing of germ cell migration. In addition, bandicoots (but not opossums) are the only marsupial group with fully invasive allantoic placentation, which could influence the preferred route of migration.

Many of the PGCs were migrating in groups, rather than singly, in the central area of the dorsal mesentery between days 22 and 24 after RPY. Similar observations of grouped, mitotic and migrating PGCs have been made in the brushtail possum in the proximal region of the gonadal primordium (Ullmann, 1996). In the light of recent observations in the mouse that germ cells link up with each other by long cellular processes forming extensive networks during migration (Gomperts $e t$ al., $1994 a, b)$, it is likely that the groups of PGCs observed in tammar fetuses are similarly linked. Such aggregations into closely apposed masses appear to be an important component of PGC migration (Gomperts et al., 1994a). Although most PGCs appeared to migrate to the gonad, a few were observed in sites such as the adrenal and mesonephros. Such ectopic germ cells have also been observed in eutherian mammals (Zamboni and Upadhyay, 1983) and may reflect an error in the mechanisms that normally guide migration. The fate of the PGCs observed in the embryonic neurectoderm is unknown.

The increase in the germ cell population in embryos between days 21 and 25 can be accounted for by three consecutive mitotic divisions of the PGCs, giving a generation time of about $24-30 \mathrm{~h}$, which is longer than that suggested for mice (Lawson and Hage, 1994). The estimated 4000 germ cells present on day 25, 1 day before parturition, are somewhat 
fewer than the 26000 reported for the mouse on day 13.5 after coitus (Tam and Snow, 1981). However, germ cells continue to multiply mitotically postpartum in the tammar and do not reach peak numbers, or complete the switch from mitosis to meiosis, until 50 days after birth (Alcorn and Robinson, 1983).

Thus, germ cell migration in the tammar is similar to that in the mouse except that the hindgut route appears to be avoided. The tammar has many advantages for the study of PGC biology in a mammal because the primitive streak develops on the surface of the vesicle rather than in the complicated egg cylinder of the mouse. The earliest stage at which we could estimate the number of ALP-positive cells was in a 13-14somite embryo (around day 17) where there were around 80-90 presumptive PGCs in three groups, two lateral and one at the posterior of the dorsal nerve cord. This is slightly fewer than the 125 PGCs found in the early-somite mouse embryo 8 days postcoitum (Ginsburg et al., 1990). Although in gross morphology the tammar embryo is superficially like that of the chick and much less complicated than the mouse, it is a mammal. Thus, it may be possible to define the origin and signals determining mammalian PGC migration further using specific markers in bilaminar and early trilaminar marsupial embryos.

We thank A. Duns, R. Moyle, J. Clark, B. Abaloz and D. Paul for excellent technical assistance. S. L. Ullmann is grateful for hospitality in the Zoology Department, University of Melbourne, and for travel grants received from the Royal Society, London. This work was supported by grants from the National Health and Medical Research Council of Australia to M. B. Renfree, G. Shaw and R. V. Short.

\section{References}

Abercrombie M (1946) Estimation of nuclear population from microtome sections Anatomical Record 94 239-247

Alcorn GT (1975) The development of the ovary and urogenital ducts in the tammar wallaby, Macropus engenii (Desmarest, 1817) PhD thesis, Macquarie University, New South Wales

Alcorn GT and Robinson ES (1983) Germ cell development in female pouch young of the tammar wallaby (Macropus eugenii) Journal of Reproduction and Fertility 67 319-325

Byskov AG and Hoyer PE (1994) Embryology of mammalian gonads and ducts. In The Physiology of Reproduction (2nd Edn) pp 487-540 Eds E Knobil and JD Neill. Raven Press, New York

Gardner RL (1978) The relationship between cell lineage and differentiation in the early mouse embryo. In Genetic Mosaics and Cell Differentiation pp 205-242 Ed. WJ Gehring. Springer-Verlag, Berlin

Ginsburg M (1994) Primordial germ cell migration in birds. In Germline Development (Ciba Foundation Symposium 182) pp 52-67. Wiley, Chichester

Ginsburg M (1996) Origin of primordial germ cells in the prestreak chick embryo Developmental Genetics 19 290-301

Ginsburg M and Eyal-Giladi H (1986) Temporal and spatial aspects of the gradual migration of primordial germ cells from the epiblast into the germinal crescent in the avian embryo Journal of Embryology and Experimental Morphology 95 53-71

Ginsburg M and Eyal-Giladi H (1987) Primordial germ cells of the young chick blastoderm originate from the central zone of the area pellucida irrespective of the embryo-forming process Development 101 209-219
Ginsburg M, Snow MHL and McLaren A (1990) Primordial germ cells in the mouse embryo during gastrulation Development 110 521-528

Gomperts M, Garcia-Castro M, Wylie C and Heasman J (1994a) Interactions between primordial germ cells play a role in their migration in mouse embryos Development 120 135-141

Gomperts M, Wylie C and Heasman J (1994b) Primordial germ cell migration. In Germline Development (Ciba Foundation Symposium 182) pp 121-139. Wiley, Chichester

Hughes RL and Hall LS (1988) Structural adaptations of the newborn marsupial. In The Developing Marsupial. Models for Biomedical Research pp 8-27 Ed. CH Tyndale-Biscoe and PA Janssens. Springer-Verlag, Berlin Heidelberg

Lawson KA and Hage WJ (1994) Clonal analysis of the origin of primordial germ cells in the mouse. In Germline Development pp 68-91 (Ciba Foundation Symposium 182). Wiley, Chichester

McLaren A (1981) Germ Cells and Soma Yale University Press, New Haven and London

Maitland P and Ullmann SL (1993) Gonadal development in the opossum Monodelphis domestica. The rete ovarii does not contribute to the steroidogenic tissues Journal of Anatomy 183 43-56

Nieuwkoop PD and Sutasurya LA (1979) Primordial Germ Cells in the Chordates Cambridge University Press, Cambridge, U.K

Nieuwkoop PD and Sutasurya LA (1981) Primordial Germ Cells in the Invertebrates From Epigenesis to Preformation Cambridge University Press, Cambridge

O W-S, Short RV, Renfree MB and Shaw G (1988) Primary genetic control of somatic differentiation in a mammal Nature 331 716-717

Renfree MB (1973) The composition of fetal fluids of the marsupial Macropus eugenii. Developmental Biology $3262-79$

Renfree MB and Tyndale-Biscoe CH (1973) Intrauterine development after diapause in the marsupial Macropus eugenii. Developmental Biology 32 28-40

Renfree MB and Tyndale-Biscoe CH (1978) Manipulation of marsupial embryos and pouch young. In Methods in Mammalian Reproduction pp 307-331 Ed. JC Daniel. Academic Press, New York

Renfree MB, Fletcher TP, Blanden DR, Lewis PR, Shaw G, Gordon K, Short RV, Parer-Cook E and Parer D (1989) Physiological and behavioural events around the time of birth in macropodid marsupials. In Kangaroos, Wallabies and Rat Kangaroos pp 323-337 Eds P Jarman, D Hume and G Grigg. Surrey Beatty and Sons, Sydney

Renfree MB, O W-S, Short RV and Shaw G (1996) Sexual differentiation of the urogenital system in a marsupial, the tammar Macropus engenii. Anatomy and Embryology 194 111-134

Snow MHL (1981) Autonomous development of parts isolated from primitive streak-stage mouse embryo. Is development clonal? Journal of Embryology and Experimental Morphology 65 269-287

Tam PPL and Snow MHL (1981) Proliferation and migration of primordial germ cells during compensatory growth in mouse embryos Journal of Embryology and Experimental Morphology 64 133-147

Tyndale-Biscoe CH and Renfree MB (1987) Reproductive Physiology of Marsupials Cambridge University Press, Cambridge

Ullmann SL (1981) Observations on the primordial germ cells of bandicoots (Peramelidae; Marsupialia) Journal of Anatomy 132 581-595

Ullmann SL. (1989) Ovary development in bandicoots: sexual differentiation to follicle formation Journal of Anatomy 165 45-60

Ullmann SL (1993) Differentiation of the gonads and initiation of mammary gland and scrotum development in the brushtail possum Trichosurus vulpecula (Marsupialia) Anatomy and Embryology 187 475-484

Ullmann SL (1996) Development of the ovary in the brushtail possum Trichosurus vulpecula (Marsupialia) Journal of Anatomy 189 651-665

Wei G and Mahowald AP (1994) The germ line: familiar and newly uncovered properties Annual Review of Genetics 28 309-324

Wreford NG (1995) Theory and practice of sterological techniques applied to the estimation of cell number and nuclear volume in the testis Microscopy Research \& Techniques 32 423-436

Zamboni L and Upadhyay S (1983) Germ cell differentiation in mouse adrenal glands Journal of Experimental Zoology 228 173-193 